\title{
Optical design of infrared pyramid wavefront sensor for the MMT
}

Shaojie Chen, Suresh Sivanandam, Siqi Liu, Jean-Pierre Veran, Phil Hinz, et al.

Shaojie Chen, Suresh Sivanandam, Siqi Liu, Jean-Pierre Veran, Phil Hinz, Etsuko Mieda, Tim Hardy, Olivier Lardiere, "Optical design of infrared pyramid wavefront sensor for the MMT," Proc. SPIE 10401, Astronomical Optics: Design, Manufacture, and Test of Space and Ground Systems, 104011H (5 September 2017); doi: 10.1117/12.2273290

EDent: SPIE Optical Engineering + Applications, 2017, San Diego, California, United States 


\title{
Optical Design of Infrared Pyramid Wavefront Sensor for the MMT
}

\author{
Shaojie Chen*a, Suresh Sivanandam ${ }^{\mathrm{a}, \mathrm{b}}$, Siqi Liu ${ }^{\mathrm{a}, \mathrm{b}}$, Jean-Pierre Veran ${ }^{\mathrm{c}}$, Phil Hinz ${ }^{\mathrm{d}}$, \\ Etsuko Mieda ${ }^{\mathrm{c}}$, Tim Hardy ${ }^{\mathrm{c}}$, Olivier Lardiere ${ }^{\mathrm{c}}$ \\ ${ }^{a}$ Dunlap Institute of Astronomy and Astrophysics, University of Toronto, 50 St. George Street, \\ Toronto ON, Canada, M5S 3H4 \\ ${ }^{\mathrm{b}}$ Department of Astronomy and Astrophysics, University of Toronto, 50 St. George Street, \\ Toronto ON, Canada, M5S 3H4 \\ ${ }^{c}$ National Research Council Herzberg Astronomy and Astrophysics, 5071 W. Saanich Rd., \\ Victoria, BC, Canada, V9E 2E7 \\ ${ }^{\mathrm{d}}$ Steward Observatory, University of Arizona, 933 N. Cherry Ave, Tucson, AZ, USA, 85721
}

\begin{abstract}
We report on the optical design of an infrared (0.85-1.8 $\mu \mathrm{m})$ pyramid wavefront sensor (IRPWFS) that is designed for the $6.5 \mathrm{~m}$ MMT telescope adaptive optics system using the latest developments in low-noise infrared avalanche photodiode arrays. The comparison between the pyramid and the double-roof prism based wavefront sensors and the evaluation of their micro pupils' quality are presented. Our analysis shows the use of two double-roof prisms with achromatic materials produces the competitive performance when compared to the traditional pyramid prism, which is difficult to manufacture. The final micro pupils on the image plane have the residual errors of pupil position, chromatism, and distortion within $1 / 10$ pixel over the $2 \times 2$ arcsecond field of view, which meet the original design goals.
\end{abstract}

Keywords: Wavefront measurement, Pyramid wavefront sensor, Pupil imaging, Adaptive optics

\section{INTRODUCTION}

The pyramid wavefront sensor (PWFS) is a novel concept, which was proposed by Ragazzoni for first time in $1996^{[1]}$. The concept of PWFS originates from the standard Foucault knife-edge, and is an effective method for adaptive optics (AO) wavefront sensing in astronomy. The historical development of PWFS is described greater detail by [Campbell \& Greenaway(2006)] ${ }^{[2]}$.

The previous work has shown that the PWFS has better sensitivity than the traditional Shack-Hartmann wavefront sensor (SHWFS) ${ }^{[1,3]}$. This improves the sensitivity of PWFSes to faint stars when compared to SHWFSs. As a result, the use of PWFSs can provide higher Strehl ratios, larger sky coverage, and higher contrast imaging capability. Some teams have already developed different types of PWFSs to test the behaviors and characteristics of PWFS ${ }^{[4-7]}$, however, there have not been detailed studies of the PWFS in the short-wave infrared (0.85-1.8 $\mu \mathrm{m})$. The benefits can be substantial where infrared PWFS can increase sky coverage and observe highly obscured regions within our galaxy.

We design an infrared $(0.85-1.8 \mu \mathrm{m})$ pyramid wavefront sensor (IRPWFS) that is developed for the 6.5m MMT telescope to improve its existing AO system. The low-noise, high frame-rate SAPHIRA IR APD image sensor is used for the detection of the high-quality micro pupils. Considering the challenges in manufacturing the four-sided pyramid prism with precise angles and a sharp tip, our design employs the double-roof prism structure as the substitute of real pyramid prism to split light into four quadrants to form micro pupils on the detector. The wavefront measurement is calculated by comparing the intensities inside four micro pupils ${ }^{[5]}$, so the micro pupils' quality determines the accuracy of wavefront measurement for the AO system directly.

In this paper, we discuss the optical design of the IRPWFS, and how the different structures of pyramid prism, including a single pyramid, double-roof, double-pyramid and two double-roof prisms affect the design. The design of the two double-roof prisms has been adopted for the PWFS after considering the competitive performance and the

sjchen@dunlap.utoronto.ca

Astronomical Optics: Design, Manufacture, and Test of Space and Ground Systems, edited by Tony B. Hull, Dae Wook Kim, Pascal Hallibert, Gary B. Hughes, Ronald G. Pirich, Proc. of SPIE Vol. 10401, 104011H · @ 2017 SPIE · CCC code: 0277-786X/17/\$18 · doi: 10.1117/12.2273290 
challenges in manufacturing the pyramid. In order to minimize the chromatic aberrations, the achromatic pair is adopted for the two double-roof prisms. After optimization, we obtained good image quality over the micro pupils, which validates our design's use for PWFS.

\section{REQUIREMENT ANALYSIS AND THEORETICAL DESIGN}

\subsection{Principle of the Pyramid Wavefront Sensing}

The sketch of the PWFS is shown in Figure 1. A transmissive pyramid prism is located on the focal plane of the telescope, which splits the beam equally into four parts. After the imaging system, four micro pupils are imaged onto the detector. The PWFS normally works dynamically, which adopts the steering mirror to modulate the focused point turning around the tip of the pyramid prism. The wavefront slope can be derived from the pixel intensity ${ }^{[5,8]}$, as described in Figure 1.

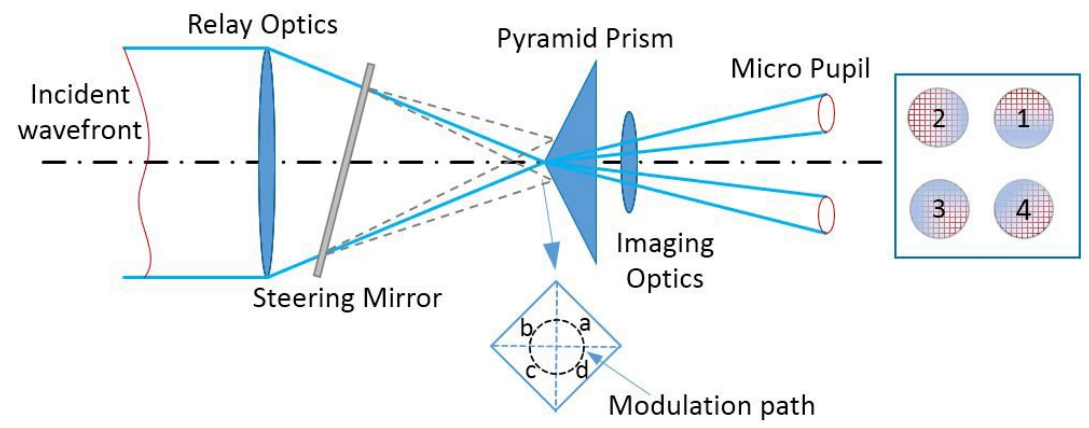

Figure 1: Sketch of the PWFS. The pyramid prism is located on the focal plane of the relay optics, which splits the beam equally into four parts. The imaging optics forms four micro pupils on the detector.

The illumination of these micro pupils depends on the wavefront errors. The signal $S$ is the difference in intensities of $I_{1}, I_{2}, I_{3}$, and $I_{4}$ between the corresponding pixel within the four pupils.

$$
\begin{aligned}
& S_{x}(x, y)=\frac{I_{1}(x, y)+I_{4}(x, y)-\left[I_{2}(x, y)+I_{3}(x, y)\right]}{I_{1}(x, y)+I_{2}(x, y)+I_{3}(x, y)+I_{4}(x, y)} \\
& S_{y}(x, y)=\frac{I_{1}(x, y)+I_{2}(x, y)-\left[I_{3}(x, y)+I_{4}(x, y)\right]}{I_{1}(x, y)+I_{2}(x, y)+I_{3}(x, y)+I_{4}(x, y)}
\end{aligned}
$$

Applying a circular modulation with a small radius, and the small wavefront errors can be calculated:

$$
\begin{aligned}
& \frac{\partial W(x, y)}{\partial x} \approx \frac{\pi V}{2 f} S_{x}(x, y) \\
& \frac{\partial W(x, y)}{\partial y} \approx \frac{\pi V}{2 f} S_{y}(x, y)
\end{aligned}
$$

Where $V$ is the modulation radius, and $f$ is the distance between steering mirror and tip of the pyramid. The wavefront errors calculation depends on the pixel intensity, which requires the pupil mapped accurately on the detector.

\subsection{Top-Level Requirements}

This IRPWFS is designed for MMT, which is a $6.5 \mathrm{~m}$ telescope located on the summit of Mt. Hopkins. The secondary mirror of the MMT is a deformable mirror which is made of the thin glass shell with $2 \mathrm{~mm}$ thick and $640 \mathrm{~mm}$ in diameter deformed by over 300 vice coils. The current wavefront sensing of MMT is implemented by the SHWFS, and our PWFS upgrade will be more sensitive to fainter stars and offer larger sky coverage. The list of the top-level requirements is given in Table 1. 
Table 1: Requirement of IRPWFS

\begin{tabular}{|c|c|}
\hline Requirement & Value \\
\hline Sub-apertures & $24 \times 24$ \\
\hline Wavelength & $0.85-1.8 \mu \mathrm{m}$ \\
\hline Field of view & $\sim 2 \operatorname{arcsec}$ (Square) \\
\hline Modulation & $\leq 5 \lambda / D$ \\
\hline Pupil image quality (static) ${ }^{*}$ & $<1 / 10$ pixel $\mathrm{rms}$ \\
\hline Pupil smearing (dynamic) ${ }^{* *}$ & $<1 / 10$ pixel rms \\
\hline Pupil jitter & $<1 / 10$ pixel \\
\hline Residual pupil chromatism & $<1 / 10$ pixel rms \\
\hline Pupil distortion & $<1 / 10$ pixel $\mathrm{rms}$ \\
\hline Detector area & $320 \times 256$, SAPHIRA IR APD array ${ }^{[9]}$ \\
\hline Pixel pitch & $24 \mu \mathrm{m}$ \\
\hline Input $\mathrm{F} / \#$ & 15 \\
\hline Frame rate & $1 \mathrm{kHz}$ \\
\hline
\end{tabular}

*: No modulation of the steering mirror;

$* *$ : Modulate the steering mirror with the angle of $5 \lambda / D$;

The IRPWFS consists of relay optics, pyramid prism, and imaging optics. The relay optics resizes the image after the telescope to the tip of the pyramid prism with the appropriate focal ratio. The relay optics contains an intermediate pupil plane, and the atmosphere dispersion corrector (ADC) and modulation mirror will be located closed to this location. The tip of the pyramid prism is located at the focal plane of the relay optics. The beam split by the pyramid prism travels through the imaging optics. Finally, four high-quality micro pupils are imaged with sufficient fidelity on a low-noise, high frame-rate infrared APD image sensor. The schematic layout of the PWFS is shown in Figure 2.

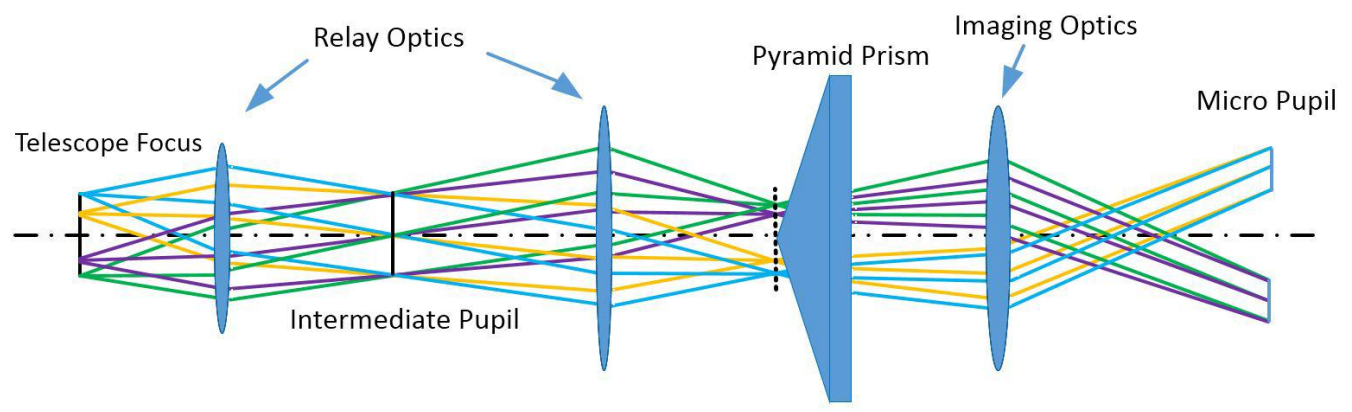

Figure 2: Schematic Layout of PWFS, which consists of relay optics, pyramid prism, and imaging optics.

The final goal of the optical system design is to achieve stable and high-quality micro pupils on the detector. Given that the PWFS does the pupil imaging, which is different from the traditional imaging system, it is important to clearly know the design constraints, and how to evaluate the quality of the micro pupils. We are going to discuss the main considerations for each optical component below.

\subsection{Relay Optics}

The relay optics reimage the object on the tip of the pyramid with the appropriate magnification. The ratio of the full width at half maximum (FWHM) of PSF to the width of the tip of the pyramid prism affects the system transmission. Due to the manufacturing limitation, the tip of the pyramid cannot be infinitely sharp. To make sure more than $50 \%$ of incoming light is transmitted in micro pupils, we design the size of the incoming PSF at the tip of the pyramid to be more than three times the width of the pyramid tip ${ }^{[10]}$. If the width of the tip is 20 microns, the FWHM of PSF at a wavelength of $1.5 \mu \mathrm{m}$ should be more than 60 microns. At the same time, we need to consider the size of the intermediate pupil in the relay optics. The output focal ratio of the telescope is F/14.62, thus the focal ratio on tip is $\mathrm{F} / 45$.

\subsection{Pyramid Prism Design}

The pyramid prism is located on the focal plane of the relay optics, which splits the beam into four parts equally, and projects the beams to the different quadrants precisely for the final micro pupils. The design of the pyramid prism is 
critical in the system, which does not only decide the quality of the micro pupils, but also drives the size and the layout of the imaging system. Moreover, we need to consider how challenging it is to manufacture the prism and align the system. To fulfill to the system requirements, the pyramid prism needs to have accurate base angles, which determine the positions of the micro pupils. It also should have equal four sides with sharp edges to guarantee the intensities of each micro pupils are equal and with little energy loss.

Theoretically, the pyramid prism can be decomposed into roof prism in two directions. We simulated four types of "pyramids", including the single pyramid, double-roof, double pyramid, and two double-roof prisms, and the layout is shown in Figure 3. Because it is not difficult to get the double-roof prism equivalent to the single pyramid prism, the two double-roof prisms should be equivalent to a double-pyramid. Given the manufacturing challenge of pyramid prism, we consider using the double-roof prism instead of the four-sided pyramid prism. Compared to the four-sided pyramid prism, the two-sided roof prism is much easier to manufacture and with higher precision. However, it comes with the trade-off that it is more challenging to align.

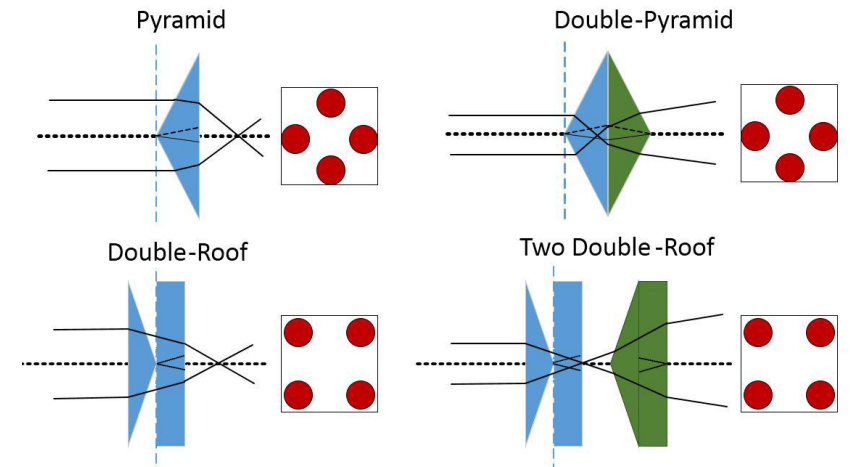

Figure 3: Different types of pyramid design, including single pyramid, double-roof, double pyramid, and two double-roof prisms.

When the "pyramid" prisms are placed in the optical path, they will cause the chromatic aberration due to the dispersion of the prism. In the case of the single pyramid (double-roof) prism, because the base angles are usually very small, they do not introduce larger chromatic aberrations. However, the chromatic aberration still cannot be compensated by the rest of the optics, and the prism with shallow angles are difficult to make. Compared to the single pyramid prism, the double-pyramid (two double-roof) prisms have steeper base angles, which are easier to manufacture, but they would cause significant chromatic aberration without a good choice of achromatic pair. We use two different glasses to correct the chromatic aberration and achieve an appropriate deflection angle in our system. The sketch of the the double-pyramid prism is shown in Figure 4.

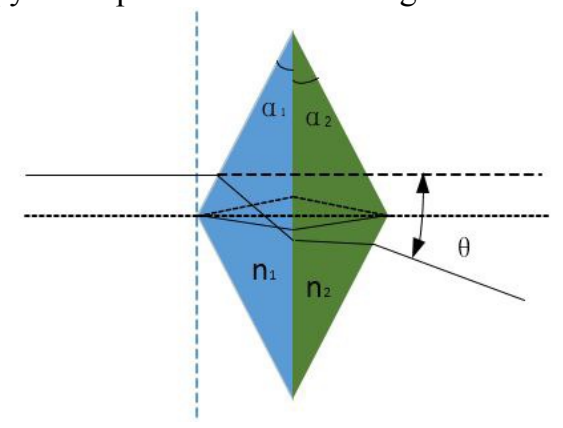

Figure 4: Sketch of double-pyramid.

The chromatic correction and the divergence angle of the double-pyramid prism can be calculated ${ }^{[10]}$ :

$$
\begin{gathered}
{\left[n_{1}\left(\lambda_{1}\right)-n_{1}\left(\lambda_{2}\right)\right] \times \alpha_{1}=\left[n_{2}\left(\lambda_{1}\right)-n_{2}\left(\lambda_{2}\right)\right] \times \alpha_{2}} \\
\theta=\left[n_{1}\left(\lambda_{c e n}\right)-1\right] \times \alpha_{1}-\left[n_{2}\left(\lambda_{c e n}\right)-1\right] \times \alpha_{2}
\end{gathered}
$$

Where $\lambda_{1}, \lambda_{2}$, and $\lambda_{\text {cen }}$ are the minimum, maximum, and center wavelengths of the system. In our design, we are supposed to find the glass pair that can works in a large wavelength range from 0.85 to $1.8 \mu \mathrm{m}$, which make our pyramid design difficult. We simulated with different materials, and found the good coupled materials for the double-pyramid prisms design, which can correct the chromatic aberration well over the whole system wavelength range and have the better performance than single prism. After we finished the double-pyramid prism design, we 
converted it to the two double-roof prisms, and found the double-roof prisms have the similar performance compared to the four-sided pyramid prism.

\subsection{Imaging Optics}

The following optical system after pyramid prism has the same input focal ratio as the output focal ratio of the relay system, because the pyramid has no power. The pyramid prism splits the beam into four parts equally, and each part of the beam will form the micro pupil on the detector after the imaging system. The imaging system plays an important role in correcting all the imaging aberrations to achieve the required performance. In the PWFS, one of the most significant aberration is the residual aberration due to the dispersion of the pyramid prism, which has to be compensated by the imaging optics. This requires the imaging system to generate the appropriate inverse chromatic aberration over the wide wavelength range from $0.85-1.8 \mu \mathrm{m}$. Even though, we still need to design the pyramid prism to make the chromatic aberration as small as possible.

\subsection{Parameters of IRPWFS}

Based on the above analysis, we calculated the first order optical parameters for the IRPWFS, listed in table 2.

Table 2: First order Optical Parameters of IRPWFS

\begin{tabular}{|l|l|}
\hline Wavelength range & $0.85-1.8 \mu \mathrm{m}$ \\
\hline Field of view & $2 \times 2 »$ \\
\hline Input F/\# & $\mathrm{F} / 14.62$ \\
\hline F/\# on pyramid tip & $\mathrm{F} / 45$ \\
\hline Magnification of relay optics & 3.02 \\
\hline Relay optics & Imaging Telecentric \\
\hline Material pair of double-pyramid (two double-roofs) & $\mathrm{N}-\mathrm{SK} 11$ \& Fused Silica \\
\hline Base angles of double-pyramid (two double-roofs) & $15.06 / 15.80$ degree \\
\hline Clear aperture of roof & $10 \mathrm{~mm}$ \\
\hline Thickness of each roof & $5 \mathrm{~mm}$ \\
\hline Distance between two double-roofs & $10 \mathrm{~mm}$ \\
\hline Focal length of imaging system & $25.43 \mathrm{~mm}$ \\
\hline Diameter of micro pupil & $0.576 \mathrm{~mm}, 24$ Pixels \\
\hline Separation of micro pupil center & $48 \mathrm{Pixels}$ \\
\hline Detector pixels required & $72 \times 72 \mathrm{Pixels}$ \\
\hline
\end{tabular}

\section{System Optical Design}

According to the paraxial analysis, we designed the actual system with Zemax shown in Figure 5. For a simplified view of the IRPWFS, we do not show the system folds.

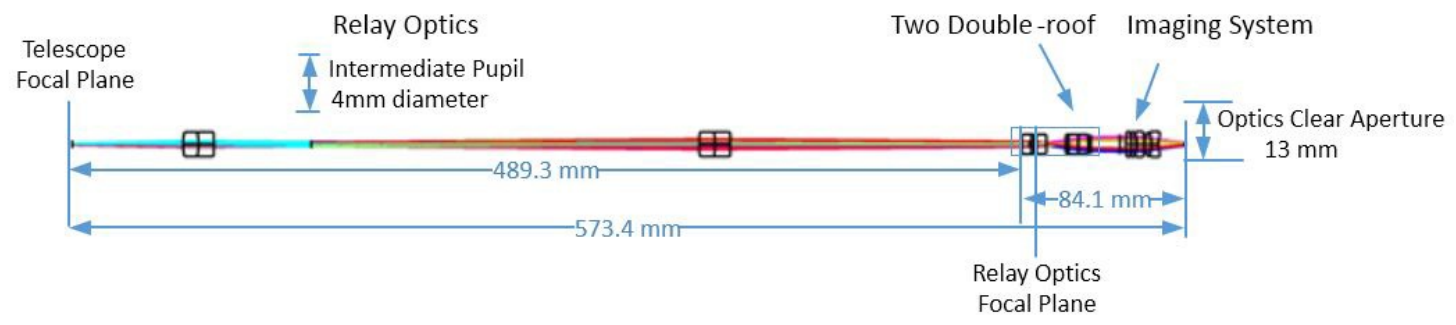

Figure 5: Layout of the IRPWFS. It consists of relay optics, two double-roof prisms, and an imaging system.

\subsection{Relay Optics Design}

We use two doublets to achieve the telecentric relay optics design with good imaging quality on the tip of the first double-roof prism. The achromatic doublet consists of CaF2 and S_FTM16 with the focal length of $58.38 \mathrm{~mm}$ and $176.81 \mathrm{~mm}$ respectively. The length of the relay optics is $489.3 \mathrm{~mm}$, and the intermediate pupil size is $4.0 \mathrm{~mm}$, which accommodates an ADC and a modulation mirror.

The image space telecentric relay optics make all different field of views have the same entrance angle on the tip of the double-roof prism, which makes the calculation of the two double-roof easier with good image quality, and has a loose constraint on the distance between the double-roof and imaging system. We attempted to design the double- 
roof prisms with a diverging beam, but we did not find a competitive achromatic prism pair to achieve the micro pupils with required quality.

\subsection{Two Double-roof Design}

As discussing in Section 2.4, we decided to use two double-roof prisms as the pyramid prism in IRPWFS. We simulated the double pyramid with different glasses, and chose the N_SK11 and F_Silica as the material pair with the appropriate base angles of $15.06^{\circ}$ and $15.80^{\circ}$, respectively. Following the method mentioned in Section 2.4 , we convert the double-pyramid prism to two double-roof prisms. In order to split the beam on the tip of the first doubleroof prism equally, the first double-roof prism has the same base angle of $15.06^{\circ}$ for both of the front and rear roof prisms. Because the optical paths are difference between the pyramid and double-roof prisms, the second doubleroof prism was optimized to compensate the chromatic aberration over the wide wavelength range. After the optimization, the base angles of the front and rear roof prisms in the second double-roof prism are $15.77^{\circ}$ and $16.14^{\circ}$ respectively. The layout of the two double-roof prism is shown in Figure 6.
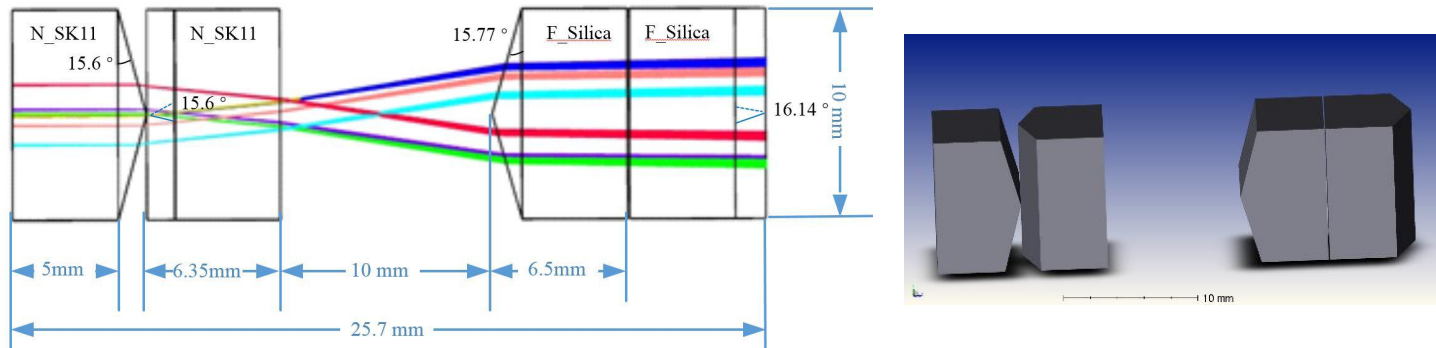

Figure 6: Design of two double-roof prisms made of N_SK11 and F_Silica, with base angles of $15.06^{\circ} / 15.06^{\circ}$ and $15.77^{\circ} / 16.14^{\circ}$.

The beam comes out from the first double-roof prism must come out from the correct surfaces on the second doubleroof prism to make the system work in the right way, thus, the two double-roof prims have to be separated appropriately. The distance between the two double-roof prisms limits the field of view of the system. In our case, the static FOV is $2 \times 2$ ", and the maximum modulation radius on the tip of the first double-roof is $5 \lambda / D$. When we do the modulation, the FOV is increased to $2.6 \times 2.6$ ". To make sure we can get the necessary FOV in the system when we include modulation, we design the distance between two double-roof prisms is $10 \mathrm{~mm}$.

\subsection{Imaging Optics Design}

The imaging optics follows the double-roof prisms, which form micro pupils on the detector. We adopt five lenses to achieve the good imaging quality. Because the imaging lens will be located in the cryogenic environment, there are no cemented lenses used, and the smallest air space is $44 \mu \mathrm{m}$ to make sure there is enough space for thermal contraction when cooling down. The glasses of the imaging system are common materials, including CaF2, SF1, NF2, and S-FTM16. After considering the limitation of packing, the distance between the last surface of the doubleroof prism and the front of the lens is $17.88 \mathrm{~mm}$, and the length from the rear of double-roof to the detector is $48.34 \mathrm{~mm}$.

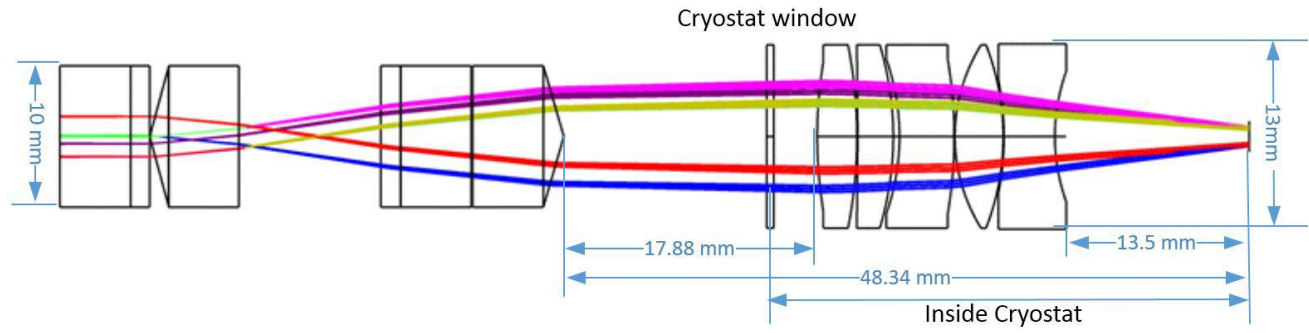

Figure 7: Layout of imaging system.

\subsection{Effects of the Steering Mirror}

In order to increase the dynamic range of the PWFS, we normally need to modulate the focused spot around the tip of the pyramid with an appropriate amplitude. In our system, we adopt the steering mirror to drive the focused point turning around the tip of the first double-roof prism with a maximum angle of $5 \lambda / D$. In order to achieve the stable performance of the PWFS in dynamic mode, it is important to analyze where the steering mirror should be located, and how the steering mirror affects the performance of the micro pupils. 
When the steering mirror is exactly located on the pupil plane, the modulation does not affect both the position and image quality of micro pupils at all. However, when the steering mirror is off the intermediate pupil plane, the modulation causes the positions of micro pupils to change on the detector. We simulated how far the steering mirror can be off from the pupil plane to be within the acceptable pupil variation.

According to the requirement, if the variation of the position of the micro pupil is less than 0.1 pixels, the offset between the steering mirror and intermediate pupil plane is accepted. The steering mirror folds the optical path with $90^{\circ}$, and the brief calculation of the maximum offset is following:

Modulation radius around the tip is: $5 \times \lambda \times F / \#=5 \times 0.0018 \times 45=0.4 \mathrm{~mm}$

The focal length of between the steering mirror and focused spot is: $f=176.8 \mathrm{~mm}$

The tilt angle along the long axis is: $\theta_{1}=\arctan \left(\frac{0.4}{176.8}\right)=0.13^{\circ}$

The tilt angle along the short axis is: $\theta_{2}=\frac{\theta_{1}}{\sqrt{2}}=0.092^{\circ}$

Magnification between the micro pupil and internal pupil is:

$$
M=\frac{d_{\text {micro pupil }}}{d_{\text {intermediate pupil }}}=\frac{24 \times 0.024}{4}=0.144
$$

The errors on the micro pupil and internal pupil are:

$$
\begin{gathered}
\Delta d_{m}=0.1 \times 24=2.4 \mu m \\
\Delta d_{i n t}=\frac{\Delta d_{m}}{M}=17 \mu m
\end{gathered}
$$

The modulation angle is $0.13^{\circ}$, and we can calculate offset distance of $\mathrm{L}$ :

$$
\begin{gathered}
\tan \left(2 \times \theta_{1}\right)=\tan \left(2 \times \frac{5 \times \lambda \times F / \#}{f}\right)=\frac{\Delta d_{\text {int }}}{L} \\
L=3.76 \mathrm{~mm}
\end{gathered}
$$

From above analysis, we can set the steering mirror off the intermediate pupil plane up to $3.76 \mathrm{~mm}$. When tilting the mirror about $\mathrm{Y}$ (long axis) and X (short axis) with $0.13^{\circ}$ and $0.092^{\circ}$ respectively, the simulation shows the focused point shifts around $0.4 \mathrm{~mm}$ to the left and up corresponding to the tilt, and the centers of micro pupils shift around $2 \mu \mathrm{m}$. In this case, the modulation causes the centers of micro pupils shifting within 0.1 pixels.

\section{SYSTEM PERFORMANCE ANALYSIS}

Evaluating the pupil performance is critical, and we created a merit function to evaluate the pupil image quality by ZPL. In the merit function, we constrained the actual positions of pupil to yield to the ideal positions across the FOVs and at different wavelengths. Moreover, the separation between the centers and radius of the micro pupils should take up the integral pixels, and the distortion should be less than 0.1 pixels. Assuming the system is symmetric, we used ZPL to populate the Merit Function Operands (MFO) of REAX and REAY to compute the real ray positions of the micro pupil in the first quadrant. When the micro pupil in the first quadrant achieves the good performance, the rest three micro pupils also obtain the similar performance. The micro pupils are arranged on the imaging plane as shown in Figure 8. 


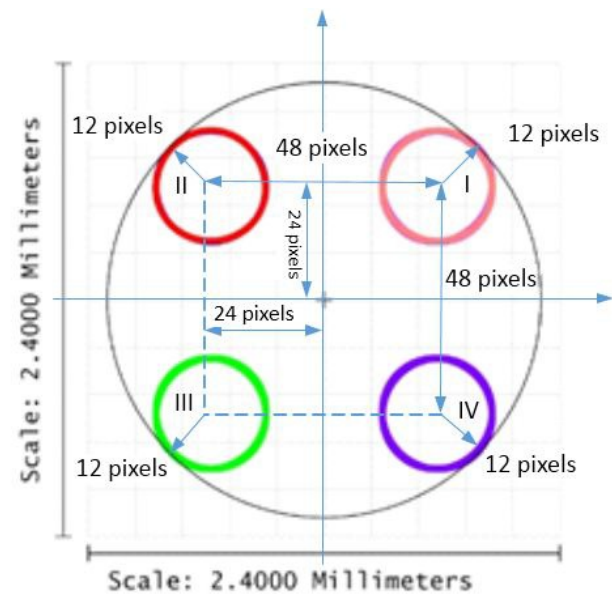

Figure 8: Micro pupils on imaging plane. The radius is 12 pixels, the separation of the centers of adjacent micro pupils is 48 pixels, and each micro pupil take up integral pixels.

In order to evaluate the image quality of micro pupils sufficiently, we calculate the spot diagram in the pupil plane. The RMS and offset of the sampling points are calculated, and the sampling points over the micro pupil are shown in Figure 9. The micro pupils in four quadrants are symmetrical, thus we take the micro pupil in the first quadrant as the example.

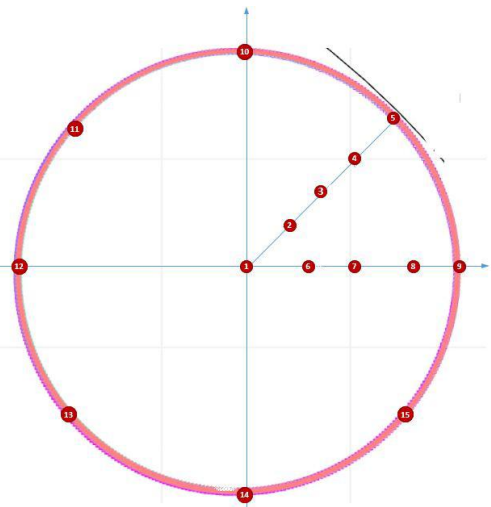

Figure 9: Sampling points on the micro pupil, 15 points are analyzed in detail.

The analysis result is shown in Figure 10. We trace the ray at a given telescope for each sampling point of micro pupil with 25 points over the science FOV at different wavelengths, which corresponds to all the blue dots in the figures. The smaller red dots are the average pupil positions over the FOV, and we consider them as actual pupil positions. The larger red dots are the ideal positions of the sampling points on the micro pupil. The red (partial) circle is centred by the paraxial position of sampling pupil point with the radius of $\mathrm{R}$. From the analysis, we find the RMS over the pupil are within $1 \mu \mathrm{m}$, which means the image quality is good. However, the offset increase along with the pupil aperture. The offset is around $4 \mu \mathrm{m}$ at the biggest distance from the pupil center, which is beyond the system requirement.
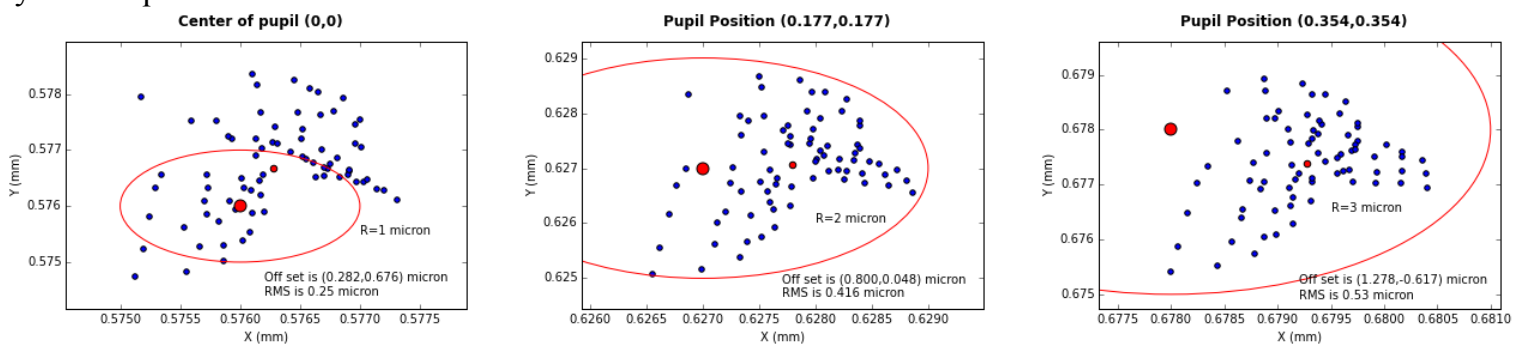

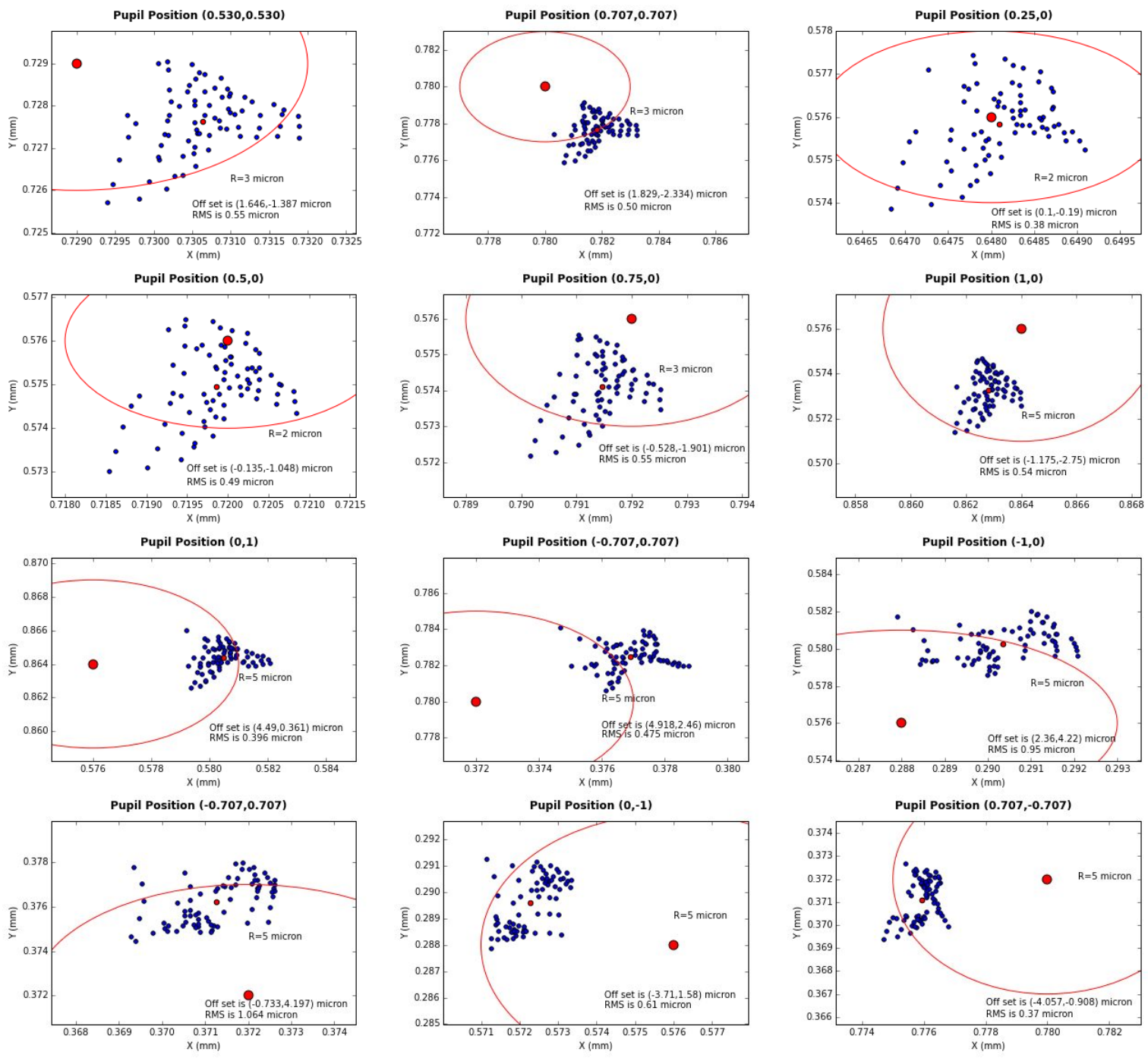

Figure 10: RMS and the offset of sampling points on micro pupil.

We did the same analysis for the micro pupils in the other three quadrants and obtained the similar results. We analyzed the error of the offset deeply, and confirm the offset is produced by the second double-roof prism within the single FOV. We mark the actual positions of the sampling points as the blue stars on the micro pupil in Figure 11 (a), and find the offset shows in terms of the rotation. The rotation of all four micro pupils is shown in Figure 11 (b).

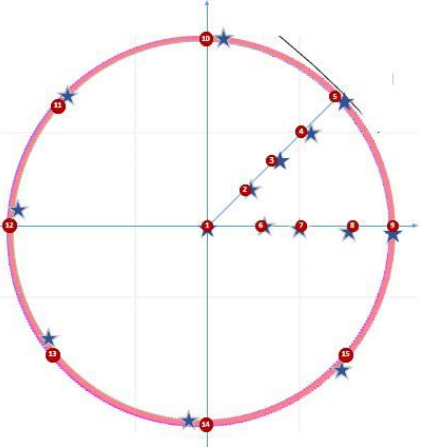

(a)

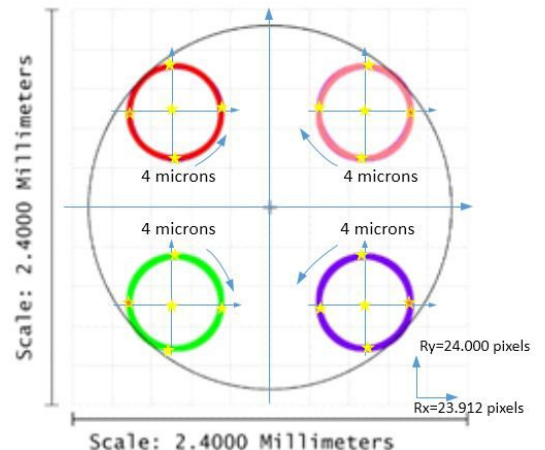

(b)

Figure 11: Rotation of micro pupil, (a) Actual and ideal pupil positions in quadrant I (b) Rotation in all four quadrants. 
In the current design, the maximum rotation error is around $4 \mu \mathrm{m}$ at the pupil edge, which is beyond the system requirement. The rotation is produced by the second double-roof prism, and it can not be corrected by the rest of optics. According to the analysis, the way to solve this problem is to decrease the base angles of the double-roof prisms to $8.0 / 8.6^{\circ}$, and increasing the focal ratio to F/99.6 on the tip of the firs double-roof prism. We have done the paraxial optical design with the new double-roof prisms, and the rotation error is less than $2 \mu \mathrm{m}$. This has the side effect of increasing the system size.

\section{SUMMARY}

This paper reports the current research status of the IRPWFS in our lab. We have finished the IRPWFS design based on the innovative double-roof prisms and done the sufficient pupil image quality evaluation. We investigated different types of "pyramid" prisms, and compared the advantages and disadvantages amongst them. After our analysis, we adopted the two double-roof prisms as the pyramid in the IRPWFS, and obtained the good material pair for the two double-roof prisms, which can correct the chromatic aberration over the wide wavelength range from 0.85 to $1.8 \mu \mathrm{m}$. The results show the two double-roof prisms can achieve very competitive performance for pupil position, chromatism, and distortion.

After the optimization by the Zemax, the system achieved good image quality pupils on the detector. In order to evaluate the pupil image quality sufficiently, we did the RMS spot size analysis of the pupil points across the micro pupils. The analysis shows the RMS of the spot diagrams is within $1 \mu \mathrm{m}$ over the whole pupil aperture, but the offset between the actual pupil point and ideal position is around $4 \mu \mathrm{m}$ on the edge of the micro pupils, which is due to the pupil's rotation. We confirmed that the rotation is caused by the second double-roof prism, which is independent of field of views. We have found the solution to solve this issue. The rotation errors decrease significantly when the roof prism is designed with the flatter angle, and the input beam has the slower focal ratio as well. We have done the paraxial optical design with the new double-roof prisms with the corresponding parameters of the system, and the rotation is controlled within $2 \mu \mathrm{m}$.

The latest simulation results suggest high tolerance on pupil positioning may not be required ${ }^{[11]}$, so our current design could still yield satisfactory performance on sky. The simulation results also loosen the mechanical tolerances on a double-pyramid prism. Experimental confirmation of the simulations is required to ensure a lower tolerance pyramid prism can be used. There are currently plans to do so.

\section{ACKNOWLEDGMENTS}

We thank the support and resources offered by the Dunlap Institute of Astronomy \& Astrophysics at University of Toronto. Shaojie Chen is supported by the Dunlap Institute for Astronomy \& Astrophysics.

\section{References:}

[1] Roberto Ragazzoni, Pupil plane wavefront sensing with an oscillating prism, Journal of Modern Optics, 199, vol. 43, No. 2, 289-293, 1996;

[2] H.I. Campbell, A. H. Greenaway, Wavefront sensing: from historical roots to the state-of-the-art, Astronomy with High Contrast Imaging III, 22, 165-185, 2006;

[3] S. Esposito, A. Riccardi, Pyramid wavefront sensor behavior in partial correction adaptive optics system, A\&A 369, L9-L12, 2001;

[4] O. Martin, J. Veran, G. Anctil, etc., Pyramidal wavefront sensor demonstrator at INO, SPIE Vol. 9148, 2014;

[5] D. Peter, M. Feldt, B. Dorner, etc., PYRAMIR: Calibration and operation of pyramid near-infrared wavefront sensor, astro-ph, 0808.0137v1, 2008;

[6] S. Esposito, A. Tozzi, D. Ferruzzi, etc., First light adaptive optics system for Large Binocular Telescope, SPIE, Vol. 4839, 2003;

[7] P. Wizinowich, M. Chun, D. Mawet, etc., Near-infrared wavefront sensing, SPIE, vol. 9909, 2016;

[8] A. Burvall, E. Daly, S. R. Chamot, C. Dainty, Linearity of the pyramid wavefront sensor, Optics Express, Vol. 14, No. 25, 2006;

[9] I. Baker, C. Maxey, L. Hipwood, K. Barnes, Leonardo (formerly Selex ES) infrared sensors for astronomy: present and future, SPIE, VII, 991505, 2016; 
[10] S. Esposito, A. Tozzi, A. Puglisi, E. Pinna, etc., Integration and test of the first light AO system for LBT, SPIE Vol. 5490, 2004;

[11] L. Wang, J-P Veran, B. Ellerbroek, Pyramid WPF performance tolerance study for NFIRAOS, AO4ELT5 conference, 2017; 